\title{
Analysis of Experimental Stand for Surface Acoustic Wave Vibrations Sensors
}

\author{
J. Filipiak ${ }^{a}$, L. SOlarz ${ }^{b}$ And G. SteCZKO ${ }^{a *}$ \\ ${ }^{a}$ Institute of Electronic and Control Systems, Technical University of Częstochowa \\ Armii Krajowej 17, 42-200 Częstochowa, Poland \\ ${ }^{b}$ Department of Civil Engineering, Military University of Technology, S. Kaliskiego 2, 00-908 Warsaw, Poland \\ The analysis of vibrations excitation in surface acoustic wave vibrations sensors is the aim of our work. The \\ sensors are parts of electronic warning system which is intended to be mounted at fences of guarded object. The \\ vibration excitation with expected acceleration is needed during experiments. Elements of fences are replaced \\ by tense strings for experimental purposes and the surface acoustic wave vibration sensors are fastened to these \\ strings. Analysis of the assembly string-sensor takes into account not only the mass of sensor but its moment \\ of inertia, too. Free vibrations of string-sensor assembly are considered. The standard excitation by pulling off \\ string in chosen point causes vibrations. The normal vibrations of assembly (frequencies, orthogonal set of normal \\ functions) are calculated and used for calculations of free vibrations. The spectrum of the standard free vibrations \\ is calculated. The results will be used in the experimental stand. The calculations enable excitation of vibrations \\ with expected spectrum. The stand enables tests of the whole warning system. The thresholds of detection and \\ reaction at concurrent intruder alarm will be investigated at this laboratory stand.
}

PACS numbers: 77.65.Dq, 68.35.Iv

\section{Introduction}

In paper [1] surface acoustic wave (SAW) vibration sensor used in linear electronic warning systems is presented. The idea of the SAW vibration sensor is illustrated in Fig. 1.

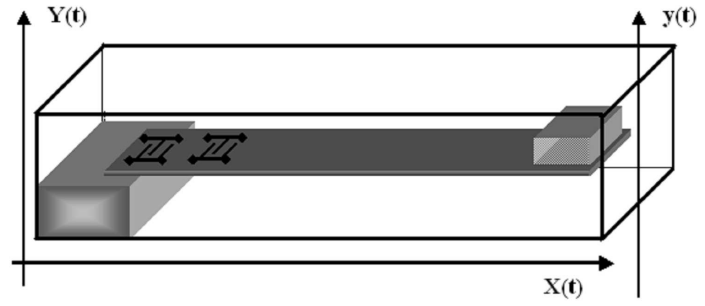

Fig. 1. Main elements of SAW vibration sensor.

One end of the plate made of anisotropic piezoelectric material is clamped and on the opposite end a mass is fixed. On the upper side of plate the SAW delay line is prepared. The delay-line works as four-terminal network. This four-terminal network may be considered as narrow-band filter with high mid-frequency (e.g. $\Omega_{0} \approx 78 \mathrm{MHz}$ ). The movement of the casing results in vibrations of the plate and as the consequence, in the delay of the line changes and the phase of signal going through the delay-line, too. The frequency of phase change is equal to the

* corresponding author; e-mail: gem@gemsc.com.pl resonant frequency of plate. Vibrations with the first resonant frequency prevail and this frequency enables distinction which sensor is activated by an intruder. The amplitude of phase changes proportionally to the amplitude of plate vibrations which depends on intensity of fence excitation. This effect is the most important for threshold vibrations sensors.

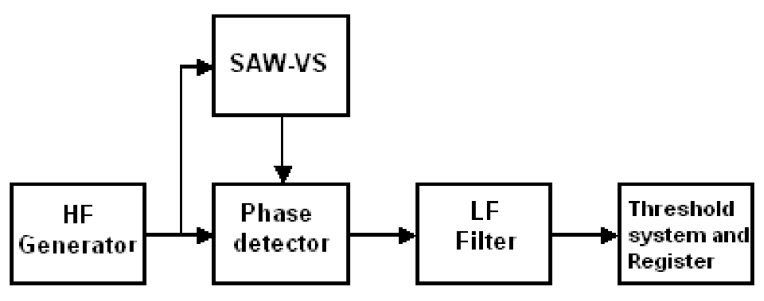

Fig. 2. The element of warning system with SAW vibrations sensor.

Direct measurement of phase shift and its frequency is rather difficult. The system seen in Fig. 2 changes the above mentioned signal into alternating signal. The alternating signal frequency is equal to the frequency of plate and its amplitude is proportional to the amplitude of phase shift in delay-line. The system seen in Fig. 2 is a four-terminal network. Such systems may be cascade-connected with another four-terminal networks having the same mid-frequency but different frequencies of plates. This enables building large systems. Therefore, measuring the amplitude of delay in SAW delay-line we 
measure the fence vibrations; mesuring frequency of delay in SAW delay-line we are able to distinguish, which of SAW vibration sensors is excited by an intruder. All sensors in the system must have different frequency of their plates.

Before site investigations, the laboratory experiments are necessary. The laboratory stand should enable concurrent excitation of some of sensors in the system with known intensity and spectrum. We propose a system with the sensors mounted at bass strings. Low frequency of excitation is expected in real fences. The stand enables investigation of interference between different signals from different sensors. Mechanical vibrations of tensile strings with mounted sensors are to be investigated. The assembly string-sensor should be excited in simple and standard way. Relations between point and size of pulling off and spectrum of free vibrations are calculated. The mass of sensor is of the same order as the mass of the string and its moment of inertia also influences vibrations. The derived orthogonality formula seems to be unknown in papers. The results of simplified theory of plate vibrations is described in Sect. 2. The discrepancy between the simplified theory and more precise one were investigated in [2]. At the base of the consideration presented in this paper more precise formula may be derived, too. The analysis of assembly string-sensor vibration is presented in Sect. 3. Some results and formulae end our considerations.

\section{Phenomena in SAW vibration sensor}

The phenomena are described using the model with one degree of freedom. HF signal transmitted by vibrating sensor changes the phase by

$$
\varphi_{n}=2 \pi \Omega_{0} \Delta \tau_{n},
$$

where $\Omega_{0}$ is for angular frequency of high frequency (HF) signal and $\Delta \tau_{n}$ is for delay-time change of the " $n$ " sensor. The delay-time change is caused by:

- change of SAW velocity produced by stresses in the vibrating plate,

- change of distance between SAW transducers also caused by vibrations.

The precise model of the phenomenon, taking into the account complicated state of deformation and many degrees of freedom, is described by the system of equations which may be solved only by numerical methods. To avoid these calculations we propose the simplified model of the plate vibrating with one degree of freedom derived by the Rayleigh method and verified by our experiments [2-6].

The delay-time change $\Delta \tau_{n}$ is proportional to the displacement of plate end during vibrations

$$
\Delta \tau_{n} \sim y(t) .
$$

From (2.1) we derive $\varphi_{n} \sim y(t)$.

The displacement of plate end is derived from the equation $[5,6]$ :

$$
\frac{\mathrm{d}^{2} y(t)}{\mathrm{d} t^{2}}+\omega_{0}^{2} \tau \frac{\mathrm{d} y(t)}{\mathrm{d} t}+\omega_{0}^{2} y(t)=\frac{\mathrm{d}^{2} Y(t)}{\mathrm{d} t^{2}},
$$

where $\frac{\mathrm{d}^{2} Y(t)}{\mathrm{d} t^{2}}$ is for the casing acceleration in $y$-direction, $\tau$ is for equivalent damping coefficient [2], $\omega_{0}$ is for resonant angular frequency of plate

$$
\omega_{0}=3.5172\left(\frac{h}{l^{2}}\right) \sqrt{\frac{E_{\mathrm{e}}}{12 \rho}}(1+r 3.9689)^{-0.5} .
$$

$l$ is the length of plate, $h$ is the thickness of plate, $\rho$ is the mass density of plate, $r$ is the ratio of the mass at the end of plate to the mass of plate, $E_{\mathrm{e}}$ is the equivalent Young modulus of plate [2]. The solution to Eq. (2.4) is known as the Duhamel integral

$$
\begin{aligned}
& y(t)=A \exp \left(-0.5 \omega_{0}^{2} \tau t\right) \sin \left(\omega_{r}(t+\alpha)\right) \\
& +\frac{1}{\omega_{r}} \int_{0}^{t} \frac{\mathrm{d}^{2} Y(\zeta)}{\mathrm{d} \zeta^{2}} \exp \left(-0.5 \omega_{0}^{2} \tau(t-\zeta)\right) \\
& \times \sin \left(\omega_{r}(t-\zeta)\right) \mathrm{d} \zeta,
\end{aligned}
$$

where

$$
\omega_{r}=\omega_{0} \sqrt{1-0.25 \omega_{0}^{2} \tau^{2}} .
$$

The constants $A$ and $\alpha$ are calculated using initial conditions.

Both parts of solution (2.6) contain the function

$$
y_{s}(t)=\exp \left(-0.5 \omega_{0}^{2} \tau t\right) \sin \left(\omega_{r} t\right)
$$

known as the impulse response. The function (2.8) is the product of the harmonic function and the exponentially diminishing function of time.

The resonant angular frequency of plate is contained in this harmonic function. The change of HF signal $\left(\Omega_{0}\right)$ is the sum of:

- convolution of impulse response and casing acceleration,

- damped free vibrations of the plate.

Both terms contain the harmonic function of time. The angular frequency is equal to the resonant angular frequency of " $n$ "-th plate. The amplitude is slowly changing with time.

If the frequencies of different plates are not the same, the system enables identification of vibrating sensor or sensors. The alarm state may be signaled when the amplitude of vibration is greater than the threshold value. If the system would register both values (frequency and amplitude) it may be used as element of guarding arrangement.

\section{Vibration of laboratory stand}

The vibrations of laboratory stand generate the casing acceleration $\frac{\mathrm{d}^{2} Y(t)}{\mathrm{d} t^{2}}$. The SAW vibration sensor mounted at string is seen in Fig. 3. The sensor vibrations are ex- 


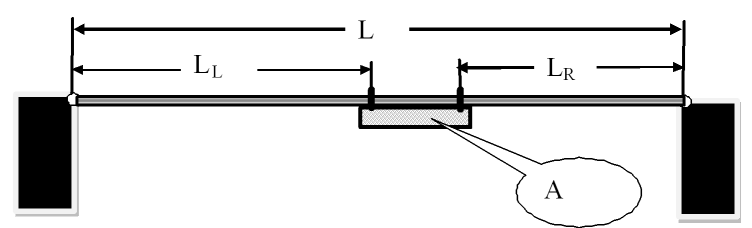

Fig. 3. The SAW vibration sensor mounted at the string.

cited in the standard and planed manner. The presented considerations enable us to plan the spectrum of vibrations.

\subsection{Mechanical parameters of the string-sensor object}

The mechanical parameters of the string-sensor object are the following:

Dimensions:

- The length of left (right) part of string $L_{\mathrm{L}}\left(L_{\mathrm{R}}\right)$, the length of string $L$.

- The area of cross-section of string $S$.

- The distance of sensor's centre of mass from the left fix-point $\chi_{\mathrm{CA}}$.

- The distance between the string and the centre of mass $d_{\mathrm{CA}}$.

- The distance between the point where the string is pulled off and the left end of string $L_{\mathrm{F}}$.

- The maximum of pull off at the initial state $z_{\mathrm{F}}$ (see Fig. 4).

Masses:

- The sensor mass $m_{\mathrm{A}}$.

- The sensor moment of inertia $I_{\mathrm{A}}=m_{\mathrm{A}} r_{\mathrm{A}}^{2}$. The symbol $r_{\mathrm{A}}$ is for sensor radius of gyration.

- The mass density of string $\rho$.

Other:

- The string tension force $N$.

- The number of harmonics $i_{\mathrm{h}}$.

During considerations the derived parameters would be useful. The list of derived parameters is the following:

- $a=\sqrt{n / \rho S}$, the wave velocity in string.

- $m_{\mathrm{w}}=\frac{m_{\mathrm{A}}}{\rho S L}$, the ratio sensor mass to string mass.

- $\lambda_{\mathrm{C}}=\frac{x_{\mathrm{CA}}}{L-L_{\mathrm{L}}-L_{\mathrm{R}}} ; L-L_{\mathrm{L}}-L_{\mathrm{R}}$ - the distance between points where the sensor is fixed to string.
- $I_{\mathrm{w}}=m_{\mathrm{w}}\left(\frac{r_{\mathrm{A}}}{x_{\mathrm{CA}}}\right)^{2} \lambda_{\mathrm{C}}^{2}$, the dimensionless moment of inertia.

- $\Omega=\frac{\omega L}{a}$, the dimensionless angular frequency.

- $\zeta=\frac{x}{L}$, the dimensionless coordinate.

- $\zeta_{\mathrm{L}}=\frac{L_{\mathrm{L}}}{L}, \zeta_{\mathrm{R}}=\frac{L_{\mathrm{R}}}{L}, \zeta_{\mathrm{F}}=\frac{L_{\mathrm{F}}}{L}$,

- $\lambda=\frac{L-L_{\mathrm{L}}-L_{\mathrm{R}}}{L}$, the ratio of the distance between points where the sensor is fixed to string to the length of string.

\subsection{Equations of the string-sensor object vibrations}

The free vibrations are considered. The argument of functions $x$ is seen in Fig. 4. The standard initial state is drawn in dashed lines.

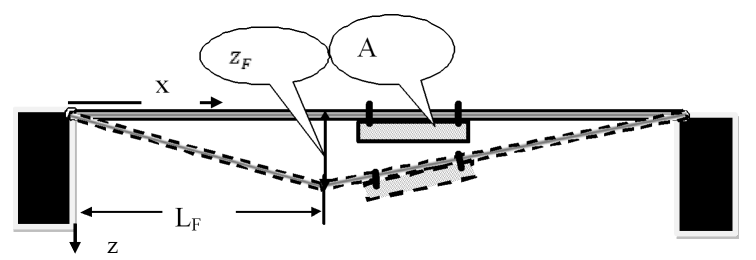

Fig. 4. The system string-sensor at standard initial conditions.

The plane, linear vibrations are considered. The symbol $z(x, t)$ is for deflection. The equation of vibrations

$$
\frac{\partial^{2} z(x, t)}{\partial t^{2}}-a^{2} \frac{\partial^{2} z(x, t)}{\partial x^{2}}=0
$$

is fulfilled for $x \in\left(0, L_{\mathrm{L}}\right)$ and $x \in\left(L-L_{\mathrm{P}}, L\right)$.

The boundary condition consists of

$$
z(0, t)=0, \quad z(L, t)=0,
$$

and equations of small, plane vibration of sensor A:

$$
\begin{aligned}
& m_{\mathrm{w}}\left(1-\lambda_{\mathrm{C}}\right) \frac{\partial^{2} z\left(L_{\mathrm{L}}, t\right)}{\partial t^{2}}+\frac{a^{2}}{L} \frac{\partial}{\partial x} z\left(L_{\mathrm{L}}, t\right) \\
& +m_{\mathrm{w}} \lambda_{\mathrm{C}} \frac{\partial^{2} z\left(L-L_{\mathrm{R}}, t\right)}{\partial t^{2}}-\frac{a^{2}}{L} \frac{\partial}{\partial x} z\left(L-L_{\mathrm{R}}, t\right)=0,
\end{aligned}
$$

$$
\begin{aligned}
& -I_{\mathrm{w}} \frac{\partial^{2} z\left(L_{\mathrm{L}}, t\right)}{\partial t^{2}}-\frac{a^{2}}{L}\left[\lambda_{\mathrm{C}} \frac{\partial}{\partial x} z\left(L_{\mathrm{L}}, t\right)+\frac{z\left(L_{\mathrm{L}}, t\right)}{L-L_{\mathrm{L}}-L_{\mathrm{R}}}\right] \\
& +I_{\mathrm{w}} \frac{\partial^{2} z\left(L-L_{\mathrm{R}}, t\right)}{\partial t^{2}}+\frac{a^{2}}{L}\left[\frac{z\left(L-L_{\mathrm{R}}, t\right)}{L-L_{\mathrm{L}}-L_{\mathrm{R}}}\right. \\
& \left.-\left(1-\lambda_{\mathrm{C}}\right) \frac{\partial}{\partial x} z\left(L-L_{\mathrm{R}}, t\right)\right]=0 .
\end{aligned}
$$

At initial state deflection is proportional to $z_{\mathrm{F}}$ and velocity is equal to 0 :

$$
z(x, 0)=z_{\mathrm{F}} U(x), \quad \frac{\partial z(x, 0)}{\partial t}=0 .
$$

The function $U(x)$ is described in (3.17). 


\subsection{Normal vibrations of the string-sensor object}

Normal vibration describes harmonic vibrations of the system with one of possible characteristic frequencies [4]. The normal functions are also calculated. The normal functions form orthogonal system. The adequate formula for inner product is disclosed. Knowing normal vibrations (frequencies, functions and inner product) we are able to calculate free and forced vibrations. We seek the normal vibrations

$$
z(x, t)=Z(\zeta) \cos (\omega t), \quad \zeta=\frac{x}{L}
$$

The function $Z(\zeta)$ is the solution of the boundary problem

$$
\begin{aligned}
& \frac{\mathrm{d}^{2} Z(\zeta)}{\mathrm{d} \zeta^{2}}+\Omega^{2} Z(\zeta)=0, \quad \Omega=\frac{\omega L}{a}, \\
& 0<\zeta<\zeta_{\mathrm{L}} \text { and } 1-\zeta_{\mathrm{R}}<\zeta<1, \quad \zeta_{\mathrm{L}}=\frac{L_{\mathrm{L}}}{L}, \\
& \zeta_{\mathrm{R}}=\frac{L_{\mathrm{R}}}{L}, \\
& Z(0)=0, \quad Z(1)=0, \\
& \frac{\mathrm{d} Z\left(\zeta_{\mathrm{L}}\right)}{\mathrm{d} \zeta}-m_{\mathrm{w}}\left(1-\lambda_{\mathrm{C}}\right) \Omega^{2} Z\left(\zeta_{\mathrm{L}}\right)-\frac{\mathrm{d} Z\left(1-\zeta_{\mathrm{R}}\right)}{\mathrm{d} \zeta} \\
& \quad-m_{\mathrm{w}} \lambda_{\mathrm{C}} \Omega^{2} Z\left(1-\zeta_{\mathrm{R}}\right)=0, \\
& \lambda_{\mathrm{C}} \frac{\mathrm{d} Z\left(\zeta_{\mathrm{L}}\right)}{\mathrm{d} \zeta}+Z\left(\zeta_{\mathrm{L}}\right)\left(\frac{1}{\lambda}-I_{\mathrm{w}} \Omega^{2}\right) \\
& \quad+\left(1-\lambda_{\mathrm{C}}\right) \frac{\mathrm{d} Z\left(1-\zeta_{\mathrm{R}}\right)}{\mathrm{d} \zeta} \\
& \quad+Z\left(1-\zeta_{\mathrm{R}}\right)\left(I_{\mathrm{w}} \Omega^{2}-\frac{1}{\lambda}\right)=0 .
\end{aligned}
$$

We seek the solution to the system (3.7)-(3.11) as

$$
Z(\zeta)= \begin{cases}B \sin (\Omega \zeta), & 0<\zeta<\zeta_{\mathrm{L}}, \\ C \sin (\Omega(1-\zeta)), & 1-\zeta_{\mathrm{R}}<\zeta<1 .\end{cases}
$$

The function (3.12) fulfils (3.7), (3.9). From (3.10), (3.11) we get

$$
\begin{aligned}
& B\left[\cos \left(\Omega \zeta_{\mathrm{L}}\right)-m_{\mathrm{w}}\left(1-\lambda_{\mathrm{C}}\right) \Omega \sin \left(\Omega \zeta_{\mathrm{L}}\right)\right] \\
& +C\left[\cos \left(\Omega \zeta_{\mathrm{R}}\right)-m_{\mathrm{w}} \lambda_{\mathrm{C}} \Omega \sin \left(\Omega \zeta_{\mathrm{R}}\right)\right]=0 \\
& B\left[\Omega \lambda_{\mathrm{C}} \cos \left(\Omega \zeta_{\mathrm{L}}\right)+\sin \left(\Omega \zeta_{\mathrm{L}}\right)\left(\frac{1}{\lambda}-I_{\mathrm{w}} \Omega^{2}\right)\right] \\
& \quad+C\left[-\Omega\left(1-\lambda_{\mathrm{C}}\right) \cos \left(\Omega \zeta_{\mathrm{R}}\right)\right. \\
& \left.\quad+\sin \left(\Omega \zeta_{\mathrm{R}}\right)\left(I_{\mathrm{w}} \Omega^{2}-\frac{1}{\lambda}\right)\right]=0 .
\end{aligned}
$$

The condition

$$
\operatorname{det}\{(3.13),(3.14)\}=0
$$

is the characteristic equation. From (3.15) we calculate characteristic (resonance) angular frequencies $\Omega_{i}$, $i=1,2,3, \ldots, i_{\mathrm{h}}$ and ratio $B_{i} / C_{i}$. The inner product of different normal functions is equal to 0 :

$$
\begin{aligned}
& \left(\Omega_{i}^{2}-\Omega_{j}^{2}\right)\left\{\left[\int_{0}^{\zeta_{\mathrm{L}}} Z_{i}(\zeta) Z_{j}(\zeta) \mathrm{d} \zeta\right.\right. \\
& \left.\quad+\int_{1-\zeta_{\mathrm{R}}}^{1} Z_{i}(\zeta) Z_{j}(\zeta) \mathrm{d} \zeta\right] \\
& \quad+m_{\mathrm{w}}\left(1-\lambda_{\mathrm{C}}\right) Z_{i}\left(\zeta_{\mathrm{L}}\right) Z_{j}\left(\zeta_{\mathrm{L}}\right) \\
& \quad+m_{\mathrm{w}} \lambda_{\mathrm{C}} Z_{i}\left(1-\zeta_{\mathrm{R}}\right) Z_{j}\left(1-\zeta_{\mathrm{R}}\right) \\
& \quad+\left(I_{\mathrm{w}}+m_{\mathrm{w}} \lambda_{\mathrm{C}}^{2}-m_{\mathrm{w}} \lambda_{\mathrm{C}}\right) \\
& \quad \times\left[Z_{i}\left(1-\zeta_{\mathrm{R}}\right)-Z_{i}\left(\zeta_{\mathrm{L}}\right)\right] \\
& \left.\quad \times\left[Z_{j}\left(1-\zeta_{\mathrm{R}}\right)-Z_{j}\left(\zeta_{\mathrm{L}}\right)\right]\right\}=0 .
\end{aligned}
$$

\subsection{Free vibrations of the string-sensor object}

The free vibrations of the system string-sensor caused by standard excitation are considered. Initial conditions (3.5) contain the function

$$
\begin{aligned}
& z(x, 0)=z_{\mathrm{F}} U(x), \\
& \quad=z_{\mathrm{F}} \begin{cases}\frac{\zeta}{\zeta_{\mathrm{F}}} & \text { for } 0 \leq \zeta \leq \zeta_{\mathrm{F}}, \\
\frac{1-\zeta}{1-\zeta_{\mathrm{F}}} & \text { for } \zeta_{\mathrm{F}}<\zeta \leq \zeta_{\mathrm{L}} \text { and } 1-\zeta_{\mathrm{R}} \leq \zeta \leq 1,\end{cases}
\end{aligned}
$$

when $L_{\mathrm{F}} \leq L_{\mathrm{L}}$,

$$
z(x, 0)=z_{\mathrm{F}} \begin{cases}\frac{\zeta}{\zeta_{\mathrm{L}}} & \text { for } 0 \leq \zeta \leq \zeta_{\mathrm{L}}, \\ \frac{1-\zeta}{1-\zeta_{\mathrm{R}}} & \text { for } 1-\zeta_{\mathrm{R}} \leq \zeta \leq 1,\end{cases}
$$

when $L_{\mathrm{L}}<L_{\mathrm{F}}<1-L_{\mathrm{R}}$,

$$
\begin{aligned}
z(x, 0) & =z_{\mathrm{F}}
\end{aligned} \begin{cases}\frac{\zeta}{\zeta_{\mathrm{F}}} & \text { for } 0 \leq \zeta \leq \zeta_{\mathrm{L}} \text { and } 1-\zeta_{\mathrm{R}} \leq \zeta \leq \zeta_{\mathrm{F}} \\
\frac{(1-\zeta)}{1-\zeta_{\mathrm{F}}} & \text { for } \zeta_{\mathrm{F}} \leq \zeta \leq 1\end{cases}
$$

when $1-L_{\mathrm{R}} \leq L_{\mathrm{F}}$. The formula (3.17) written shortly is $z(x, 0)=z_{\mathrm{F}} U(\zeta)$.

$$
\frac{\partial z(x, 0)}{\partial t}=0 \text {. }
$$

The formula (3.17) is illustrated in Fig. 5.

The free vibrations are the sum of normal vibrations [4]:

$$
z(x, t)=z_{\mathrm{F}} \sum_{i=1}^{i=i_{\mathrm{h}}} Z_{i}(\zeta) T_{i}(t) .
$$

From Eq. (3.1) we get

$\mathrm{SO}$

$$
\frac{\mathrm{d}^{2} T_{i}(t)}{\mathrm{d} t^{2}}+\omega_{i}^{2} T_{i}(t)=0, \quad \omega_{i}=\Omega_{i} \frac{a}{L},
$$

$T_{i}(t)=A_{i} \cos \left(\omega_{i} t\right)+\alpha_{i} \sin \left(\omega_{i} t\right)$,

$i=1,2,3, \ldots, i_{\mathrm{h}}$. 


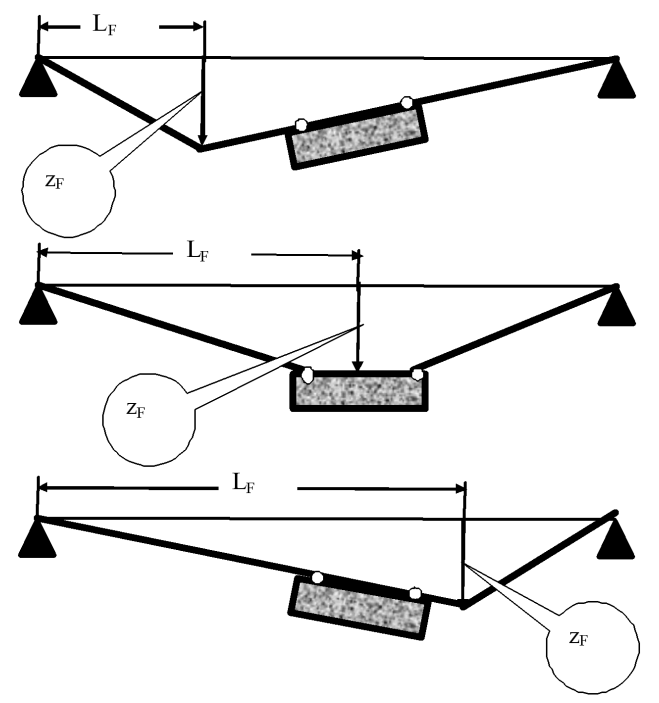

Fig. 5. The standard initial deflection of string-sensor system.

From Eq. (3.18) we get

$\alpha_{i}=0, \quad i=1,2,3, \ldots, i_{\mathrm{h}}$.

The function $U(\zeta)$ is approximated by series

$$
U(\zeta)=\sum_{j=1}^{j=i_{\mathrm{h}}} Z_{j}(\zeta) u_{j}
$$

Taking into the account (3.16) we get

$$
u_{i}=\frac{N_{i}}{F_{i i}} \quad \text { for } \quad i=1,2, \ldots, i_{\mathrm{h}},
$$

where

$$
\begin{aligned}
& N_{i}=\int_{0}^{\zeta_{\mathrm{L}}} Z_{i}(\zeta) U(\zeta) \mathrm{d} \zeta+\int_{1-\zeta_{\mathrm{R}}}^{1} Z_{i}(\zeta) U(\zeta) \mathrm{d} \zeta \\
& \quad+m_{\mathrm{w}}\left(1-\lambda_{\mathrm{C}}\right) Z_{i}\left(\zeta_{\mathrm{L}}\right) U\left(\zeta_{\mathrm{L}}\right) \\
& \quad+m_{\mathrm{w}} \lambda_{\mathrm{C}} Z_{i}\left(1-\zeta_{\mathrm{R}}\right) U\left(1-\zeta_{\mathrm{R}}\right) \\
& \quad+\left(I_{\mathrm{w}}+m_{\mathrm{w}} \lambda_{\mathrm{C}}^{2}-m_{\mathrm{w}} \lambda_{\mathrm{C}}\right) \\
& \quad \times\left[Z_{i}\left(1-\zeta_{\mathrm{R}}\right)-Z_{i}\left(\zeta_{\mathrm{L}}\right)\right] \\
& \quad \times\left[U\left(1-\zeta_{\mathrm{R}}\right)-U\left(\zeta_{\mathrm{L}}\right)\right] \\
& F_{i i}=\int_{0}^{\zeta_{\mathrm{L}}} Z_{i}(\zeta) Z_{i}(\zeta) \mathrm{d} \zeta+\int_{1-\zeta_{\mathrm{R}}}^{1} Z_{i}(\zeta) Z_{i}(\zeta) \mathrm{d} \zeta \\
& \quad+m_{\mathrm{w}}\left(1-\lambda_{\mathrm{C}}\right) Z_{i}\left(\zeta_{\mathrm{L}}\right) Z_{i}\left(\zeta_{\mathrm{L}}\right) \\
& \quad+m_{\mathrm{w}} \lambda_{\mathrm{C}} Z_{i}\left(1-\zeta_{\mathrm{R}}\right) Z_{i}\left(1-\zeta_{\mathrm{R}}\right) \\
& \quad+\left(I_{\mathrm{w}}+m_{\mathrm{w}} \lambda_{\mathrm{C}}^{2}-m_{\mathrm{w}} \lambda_{\mathrm{C}}\right)
\end{aligned}
$$

$$
\begin{aligned}
& \times\left[Z_{i}\left(1-\zeta_{\mathrm{R}}\right)-Z_{i}\left(\zeta_{\mathrm{L}}\right)\right] \\
& \times\left[Z_{i}\left(1-\zeta_{\mathrm{R}}\right)-Z_{i}\left(\zeta_{\mathrm{L}}\right)\right] .
\end{aligned}
$$

From the initial conditions (3.17) we get

$$
A_{i}=u_{i}, \quad i=1,2, \ldots, i_{\mathrm{h}} .
$$

The integrals in (3.25), (3.26) are calculated analytically (not by a numerical methods).

\section{Example of results}

Calculations enable validation of computer programs and testing the influence of parameters of the string-sensor system. The calculations would be performed for experimental research. The examples of results are presented in the paper. Calculations were carried out for constant parameters: $L=1.008 \mathrm{~m}, L-L_{\mathrm{L}}-L_{\mathrm{R}}=90 \mathrm{~mm}$, $x_{\mathrm{CA}}=50.25 \mathrm{~mm}, d_{\mathrm{CA}}=6.5 \mathrm{~mm}, S=1.2 \mathrm{~mm}^{2}$, $m_{\mathrm{A}}=27 \mathrm{~g}, r_{\mathrm{A}}=26 \mathrm{~mm}, \rho=5500 \mathrm{~kg} / \mathrm{m}^{3}$. These parameters are for the existing string and the sensor. The parameters $N=380 \mathrm{~N}, i_{\mathrm{h}}=10$, are arbitrarily chosen. The influence of parameters $L_{\mathrm{F}}$ and $L_{\mathrm{L}}$ was considered.

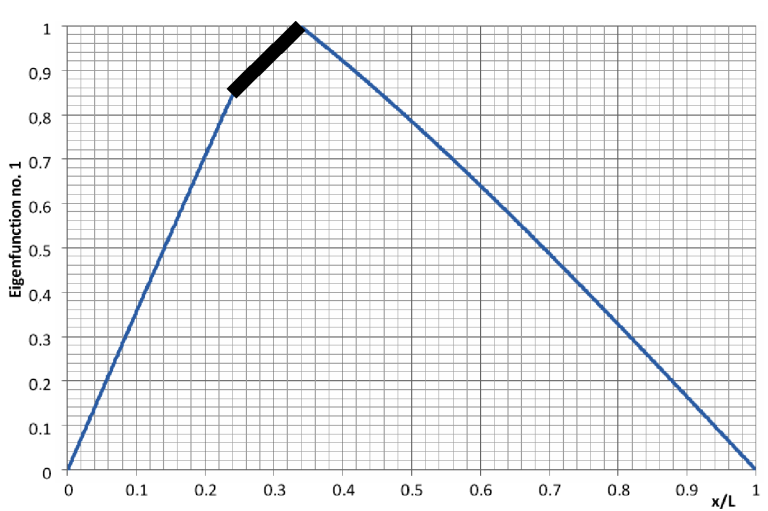

Fig. 6. Normal function no. 1. $L_{\mathrm{L}}=250 \mathrm{~mm}$.

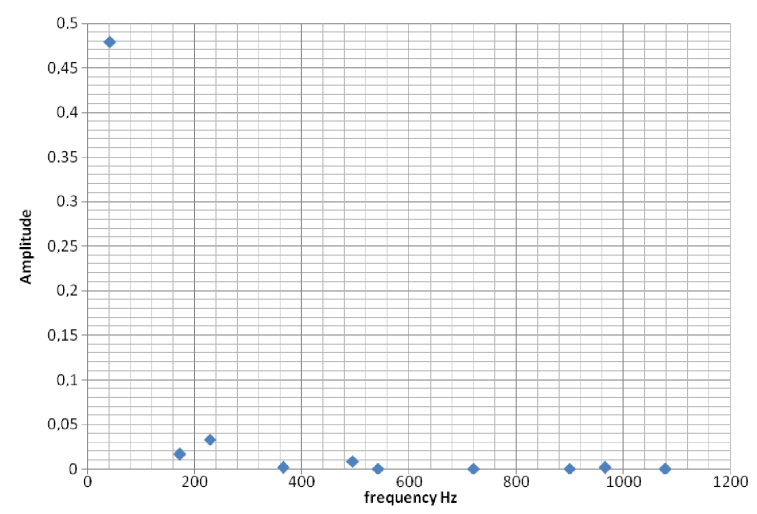

Fig. 7. Spectrum $L_{\mathrm{F}}=200 \mathrm{~mm}, L_{\mathrm{L}}=250 \mathrm{~mm}$. 


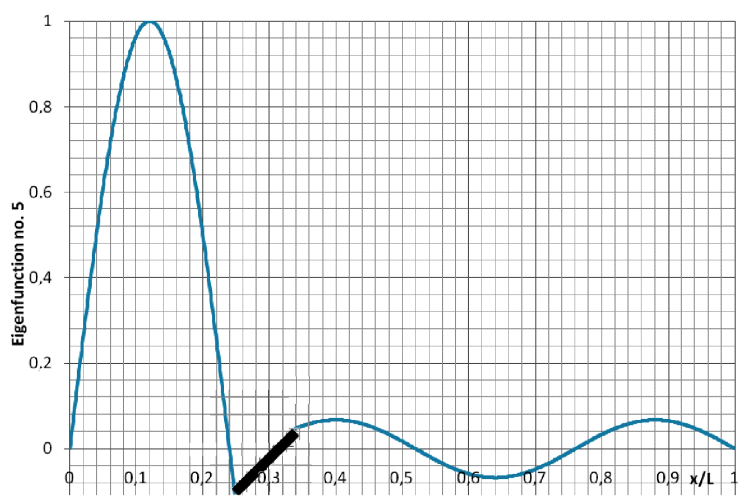

Fig. 8. Normal function no. 5. $L_{\mathrm{L}}=250 \mathrm{~mm}$.

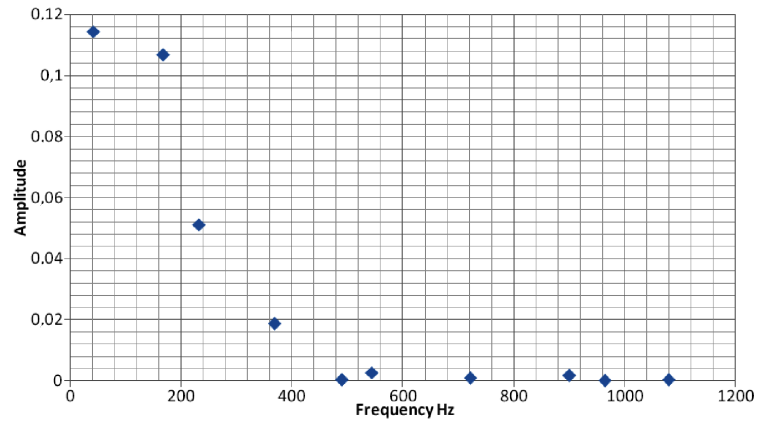

Fig. 9. Spectrum $L_{\mathrm{F}}=200 \mathrm{~mm}, L_{\mathrm{L}}=668.0 \mathrm{~mm}$.

If the shape of the standard deflection looks like the first normal function (Fig. 6), the first mode is the most important (see Fig. 8).

When the standard deflection and the first normal functions have different shape (e.g Fig. 7), then more normal modes should be taken into the considerations (see Fig. 10).

If the shape of the standard deflection looks like the first normal function, the first mode is the most important.

When the standard deflection and the first normal functions have different shape, then more normal modes should be taken into consideration.

The prepared computer programs enable computation of acceleration of the casing. More precise analysis of the sensor plate vibration is also possible thanks to knowl- edge about normal and free vibrations of the string-sensor system.

Our aim - to calculate the relations between the form of initial deflection and the spectrum of the excited free vibrations - is achieved. The program supporting experiments with linear electronic warning systems is ready.

TABLE

Normal (resonance) frequencies.

\begin{tabular}{c|c|c}
\hline \hline No. & $f[\mathrm{~Hz}]$ & Period $[\mathrm{s}]$ \\
\hline 1 & 41.7920 & 0.0239280 \\
2 & 172.937 & 0.0057825 \\
3 & 228.977 & 0.0043672 \\
4 & 365.500 & 0.0027360 \\
5 & 495.661 & 0.0020175 \\
6 & 542.838 & 0.0018422 \\
7 & 721.053 & 0.0013869 \\
8 & 900.018 & 0.0011111 \\
9 & 967.094 & 0.0010340 \\
10 & 1079.335 & 0.0009265
\end{tabular}

\section{Acknowledgments}

The work is supported as developing project "The electronic warning system with SAW vibrations detectors" of Foundation for Developing of Polish Science 2009-2011.

\section{References}

[1] J. Filipiak, L. Solarz, G. Steczko, Acta Phys. Pol. A 116, 302 (2009).

[2] J. Filipiak, L. Solarz, K. Zubko, Mol. Quant. Acoust. 25, 89 (2004).

[3] J. Filipiak, C. Kopycki, Sensors Actuators 76, 318 (1999).

[4] W. Bogusz, Z. Dżygadło, D. Rogula, K. Sobczyk, L. Solarz, in: Vibrations and Waves, Eds. S. Kaliski L. Solarz, PWN-Elsevier, New York 1992.

[5] T. Pustelny, A. Opilski, B. Pustelny, Acta Phys. Pol. A 114, A-181 (2009).

[6] J. Filipiak, SAW Acceleration Sensors, Częstochowa University of Technology Press, Częstochowa 2006 (in Polish). 